\title{
Research on the Application of Series Capacitor Compensation for Typical Medium Voltage Distribution Network
}

\author{
Ge $\mathrm{Xu}^{1, \star}$,Shiwu Xiao ${ }^{1}$, Xiaoqing $\mathrm{Xiao}^{2}$ and Likun $\mathrm{Li}^{2}$ \\ ${ }^{1}$ State Key Laboratory of Alternate Electrical Power System with Renewable Energy Sburces, North China Electrical Power University, \\ 102206 Beijing, China \\ ${ }^{2}$ Guangdong Electric Power Research Institute, 510080 Guangdong, China
}

\begin{abstract}
Series capacitor compensation can improve the operating parameters and transmission capacity of transmission line,it has been put into use in power system for many years. There is a common problem in Chinese distribution network that terminal voltage of the line is lower and instability which is caused by power supply radius being much longer than economic transmission distance and frequent load fluctuations.This paper takes the practical project as an example,emulate and analyze the advantages of the series compensation ,propose a method to determine the installing place of compensation equipment and to calculate the capacity of series capacitor,simulate the change of voltage along the line with different compensation position,series compensation degree and load ratio and compare their effects.
\end{abstract}

\section{Introduction}

Distribution network,as the last direct connection with the user's in the power supply link,is directly related to the quality of power for urban and rural users.Due to the rapid growth of electric load and the relative backwardness of distribution network construction, distribution network voltage quality is worse in recent years.It becomes an urgent and indispensable task to improve the reliability of distribution network as well as to optimize the power quality[1].As a newly developing technology,series capacitor compensation is gradually applied in the distribution line, it can improve the voltages along the radial distribution line,reduce the voltage drop at the end of the power supply line,possesses the features of real-time response and load self-adapting to voltage adjustment,especially suitable for power distribution network in remote mountain village.

In this paper,a theoretical method for calculating the capacity and compensation point of series compensated capacitor is proposed,and the effects of fixed series capacitor compensation with different compensation points,series compensation degree and load rate are analyzed and simulated for a $10 \mathrm{kV}$ radial distribution network in Xiangshan county,Shanggang.

\section{Method for calculating capacity and compensation point of series capacitor}

\subsection{Rules of selecting series capacitor installing position}

For distribution line with series compensation,the shorter the distance between compensation point and load point is, the better the effect of voltage compensation is,so selecting the position of series capacitor needs follow the rule - compensation point is as close as possible to the load point.In addition,unlike static var compensator which must be installed in the substation,it is more flexible to choose installing site,the capacitor can be added in the line near any tower at any time,and the device can be installed singly or in several places.Generally,for the short lines and intermediate lines, the device is installed at the end of the line or in the middle of the line,if the line is longer and the size of device is too large,it can be installed dispersedly in lines [2].

If the distribution line only supplies power for terminal load in a $10 \mathrm{kV}$ radial distribution network,the effect of regulating voltage has little to do with series capacitor position,the best place for compensation is the the end of the line close to the load,and the series capacitor bears the least fault stress at this time,we can get more economic protection configuration too.If there are more than one branch line along the main line,to meet the requirement of line voltage adjustment (the line voltage should be controlled to be within the range of $5 \%$ rated voltage ),it is required the voltage at the compensation point is not higher than the normal value when ensure the terminal line voltage is not low.What's more,the technical and economic nature of the series compensation and the difficult degree of its protection configuration should be considered.

At present,for centralized fixed series capacitor compensation,the common method to find installing point is select the end of the branch line as terminal point,ensure the terminal load is maximum,calculate the change of line voltage,and the point that voltage drop is half of the whole line is what we need.However,the voltage of the point often meets the voltage adjustment regulation,in this case,the method may be not a technical economic and taste.

\subsection{Method of calculating series compensation capacity}

Series compensation degree K,used to characterize the size of series compensation capacitor,is defined as the ratio of the capacitance to the equivalent reactance of the line between power supply bus and capacitor mounting point.That is:

$$
K=\frac{X_{C}}{X_{E}}
$$


$X_{C}$ is the capacitance of series capacitor,it is determined by the ratio of the maximum voltage difference needed to be compensated at the installing point to the maximum load current of the downstream line;and the node voltage of the installation depends on actual demands of compensation,for example,it can be half of the voltage drop across the line or determined by the terminal voltage.

In the formula (1-1),there are a few cases to note.The specific value of the formula may be different from specific circumstances and it should extrude the appropriate operating characteristics of the compensation device.If a distribution line only has a terminal load,without other branches along the main line, $X_{E}$ is the total line reactancee between the starting point and terminal point.If the distribution line has many branches, $X_{E}$ often refers to the equivalent reactance of all the branches before the compensation point[3].

To determine the value of $\mathrm{K}$,we should consider the actual situation of the lines and the location of device,and take account of the development level of the load after 5 years.For the distribution line, if only considering the problem of voltage regulation,the value of $\mathrm{K}$ is often taken from 1 to 4 ,and higher voltage compensation range can be obtained.

The capacity of series capacitor is determined by $\mathrm{K}$ and the normal operating current flowing through the capacitor.As the series capacitor is always in line,it will have to bear a constant overvoltage when the line runs with overload for a long time.In order to prevent the capacitor keeping sustained overvoltage,the rated current of the capacitor should be reserved for a certain margin,generally,the value of the margin is k.So,the rated current is (1-2),reference rated voltage and rated capacity can be get from (1-3),(1-4).

$$
\begin{gathered}
I_{C N}=k \cdot I_{C \max } \\
U_{C N}=X_{C N} \cdot I_{C N} \\
\mathrm{Q}_{C N}=3 I_{C N}^{2} \cdot X_{C}
\end{gathered}
$$

Taking the characteristics of load variation into account,the capacitor should have a high overload capacity bearing a higher load,the design personnel should take full account of the characteristics of the series capacitor,so as to reduce the infrastructure investment [4].

\section{Actual case analysis of $10 \mathrm{kV}$ distribution lines}

\subsection{Design and analysis of $10 \mathrm{kV}$ distribution line in Xiangshan}

The radius of $10 \mathrm{kV}$ power supply line in Zhejiang Province Xiangshan County Shanggang is 16.5 kilometers,there are total of 22 major load points in the main line and its branches,most loads are mainly gathered in the ends of the main line,the maximum load is 4.47MW,power factor at starting point is 0.91 , the total capacity of the line is $12625 \mathrm{MVA}$.

There are about 170 pole-towers in Shanggang line,the average distance of each pole-tower is 200 meters from the first tower to the thirtieth tower and 75 meters after the thirtieth,the total length is $16.5 \mathrm{~km}$.

The $10 \mathrm{kV}$ distribution line model is established,and the branch terminal load is set up with the distribution transformer at rated load.After all the parameters input,according to the maximum load running data to calculate the average load rate and adjust the whole load. The simplified model of single line diagram is shown in figure 1.

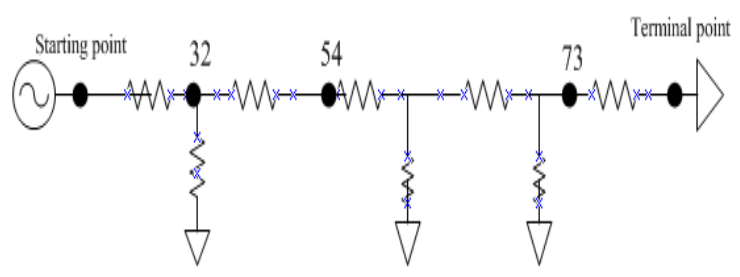

Figure 1 Simplified Model of 10kV Power Distribution line

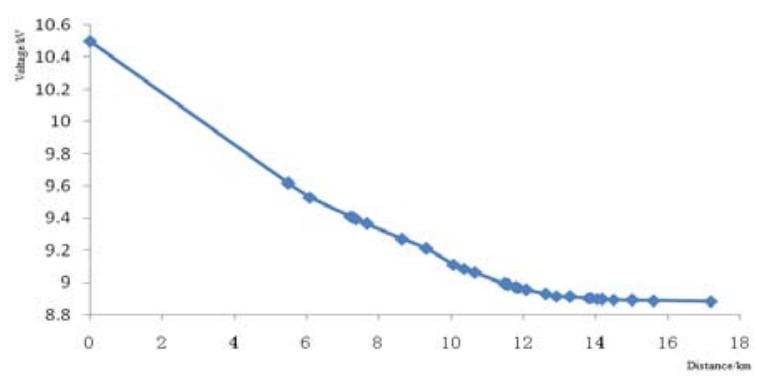

Figure 2 Voltage Curve of the Main Line

By using the program PSASP to calculate the line power flow,we get the voltage distribution along the main line and its branches without series capacitor compensation,it's shown as figure 2.In figure 2,the abscissa is distance between the starting point and the load point (all of the following voltage distribution diagrams are same),voltage sampling points are the starting,terminal point and all 22 major load points. When the power flow calculation is carried out,the voltage of the starting point is $10.5 \mathrm{kV}$, the total active power load is $4.5 \mathrm{MW}$, and average load power factor is 0.84 .

Results of power flow show active load loss of the whole line is $448 \mathrm{kw}$,and the terminal point voltage drops to $8.89 \mathrm{kV}$.The lowest voltage point in the whole network appears in the 21th tower linked to the 61th tower of the main line,and the voltage is close to $8.89 \mathrm{kV}$.

From figure 2,we can see there are two larger voltage drop gradients in all lines,one is the first long distance overhead line near the 31th tower,the other is where the load concentrated,near the 73th or 82th tower.In accordance with the above analysis,three options of the installing point are proposed :

(1) The point where voltage drops by half of the full line voltage drop,near the 32th tower of main line;

(2) The first load point without meeting the requirements of voltage ,near the 54th tower of main line; 
(3) The point where the voltage drops faster and does not meet the requirements of voltage,near the 73th or 82th tower.

Then,the effects of different compensation point,series compensation degree and load rate will be compared to find the best compensation scheme.

\subsection{Analysis of different installing position}

This section is to raise the lowest voltage in the line to the lowest voltage requirements, and do some simulations to discuss the different effects among the three options proposed.

Since series capacitor has nothing to do with line parameters behind compensation point,the trend of the voltage drop is almost the same with or without the series compensation.So we can calculate $\mathrm{K}$ by using voltage difference of compensation point and terminal point,and calculate the capacity of the capacitor(the ratio of voltage difference to the point's maximum load current)[5].The starting point voltage is $10.5 \mathrm{kV}$, the load is $4.95 \mathrm{MW}$ (a $10 \%$ increase) and the average power factor of load is still 0.84.Line voltage distribution with different compensation points is shown in figure 3,the main output data is shown in table 1.

Figure 3 shows that,after using series compensation all line voltages have a greater degree of uplift,especially the voltages of its rear line.And when all line voltages can meet the requirements of the premise,the farther the compensation position,the greater the needed series compensation degree,but also the better effect of the rear line voltage.

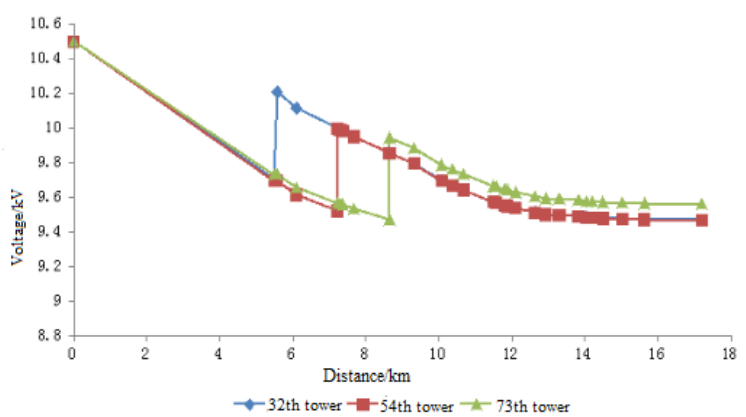

Figure 3 Voltage Distribution of Different Compensation Point

Table 1.Comparison of different compensation point.

\begin{tabular}{|c|c|c|c|}
\hline \multicolumn{4}{|c|}{ Unit: $\mathrm{kA} \backslash \mathrm{kV} \backslash \mathrm{MW} \backslash \mathrm{Mvar} \backslash$} \\
\hline $\begin{array}{c}\text { compensation } \\
\text { point }\end{array}$ & $\begin{array}{c}\text { series } \\
\text { compensation } \\
\text { capacitance }\end{array}$ & $\begin{array}{c}\text { series } \\
\text { compensation } \\
\text { degree }\end{array}$ & $\begin{array}{c}\text { active } \\
\text { load }\end{array}$ \\
\hline uncompensated & -- & -- & 4.95 \\
\hline the 32th tower & 2.85 & 1.5 & 4.95 \\
\hline the 54th tower & 5.21 & 2.1 & 4.95 \\
\hline the 73th tower & 7.16 & 2.2 & 4.95 \\
\hline
\end{tabular}

\begin{tabular}{|c|c|c|c|}
\hline $\begin{array}{c}\text { compensation } \\
\text { point }\end{array}$ & reactive load & $\begin{array}{c}\text { total reactive } \\
\text { power loss }\end{array}$ & $\begin{array}{c}\text { total } \\
\text { power } \\
\text { loss }\end{array}$ \\
\hline uncompensated & 3.215 & 1.018 & 0.581 \\
\hline the 32th tower & 3.215 & 0.832 & 0.474 \\
\hline the 54th tower & 3.215 & 0.838 & 0.479 \\
\hline the 73th tower & 3.215 & 0.82 & 0.468 \\
\hline
\end{tabular}

At the same time,comparing the data in Table 1,we can see that line power loss was reduced with series compensation, and if the compensation point is near the first unqualified voltage point,the effect of reducing power loss is less, yet if it is close to the terminal load,the effect is better.

Therefore,for the distribution line,when load is clustered at the end,it is better to set the compensation point near the load in order to obtain better voltage quality.

\subsection{Analysis of different series compensation degree}

Taking the 73th tower as the compensate point,simulate with different series compensation degree $\mathrm{K}$ and we can get different voltage distribution curves in figure 4.In figure 4,the greater the $\mathrm{K}$ is,the better the effect of enhancing the line voltage is;but with the $\mathrm{K}$ increasing,the amplitude of elevated voltage decreases relatively.That is,with the capacitance of the series capacitor becoming larger,if we improve the same rate of $\mathrm{K}$, the effect of improving the voltage will be not as good as the previous.And that means when $\mathrm{K}$ reaches a certain value if we continue to increase the it,the economic investment of compensation device will grow.

The data in Table 2 shows that with the increase of $\mathrm{K}$,the extent of reducing power loss is also decreased.Combining all of the datum,it can be inferred that if the value $\mathrm{K}$ is between 2.0 and 2.5,the effect of improving voltage and reducing line power loss is more ideal.That means the series compensation is relatively economic within the context as well as meeting the requirements of voltage regulation.

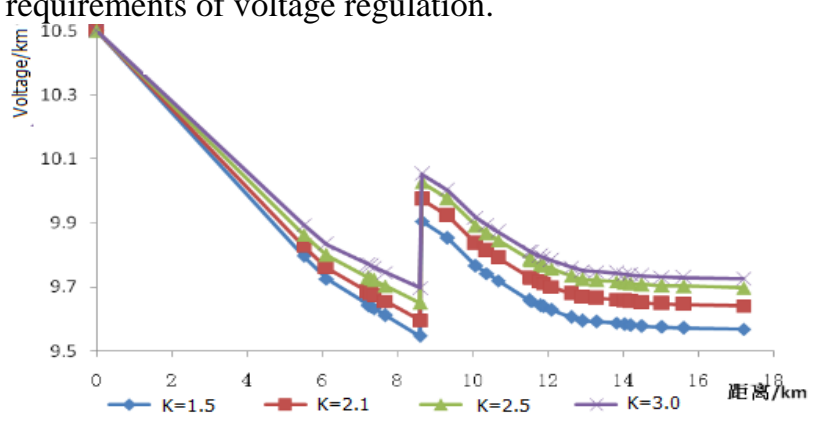

Figure 4 Voltage Distribution of Different Series Compensation Degree 
Table 2.Comparison of different series compensation degree.

\begin{tabular}{|c|c|c|c|c|c|}
\hline \multicolumn{7}{|c|}{ Unit: $\mathrm{kA} \backslash \mathrm{kV} \backslash \mathrm{MW} \backslash \mathrm{Mvar} \backslash$} \\
\hline $\begin{array}{c}\text { series } \\
\text { compensa } \\
\text { tion degr } \\
\text { ee }\end{array}$ & $\begin{array}{c}\text { series } \\
\text { compen } \\
\text { sation } \\
\text { capacit } \\
\text { ance }\end{array}$ & $\begin{array}{c}\text { voltage } \\
\text { aftive } \\
\text { load } \\
\text { of } \\
\text { termin } \\
\text { al line } \\
\text { point }\end{array}$ & $\begin{array}{c}\text { total } \\
\text { power } \\
\text { loss }\end{array}$ & $\begin{array}{c}\text { total } \\
\text { reactive } \\
\text { power } \\
\text { loss }\end{array}$ \\
\hline sated & -- & 4.5 & 8.907 & 0.448 & 0.781 \\
\hline 1.5 & 5.78 & 4.5 & 9.568 & 0.381 & 0.667 \\
\hline 2.1 & 7.16 & 4.5 & 9.641 & 0.374 & 0.653 \\
\hline 2.5 & 7.86 & 4.5 & 9.669 & 0.370 & 0.646 \\
\hline 3.0 & 10.28 & 4.5 & 9.724 & 0.362 & 0.633 \\
\hline
\end{tabular}

\subsection{Analysis of different load rate}

For medium voltage distribution lines,especially in the vast rural areas,there is a large difference between peak and valley value of load,in order to avoid that series capacitor causes line voltage too high with light load,the capacitor should be removed,if necessary[6].In this section,the loads are reduced in the same proportion,and the variation of line voltage is shown in Figure 5.

As shown in Figure 5, when $\mathrm{K}$ is determined,the voltage of the compensation point is almost determined.With the decrease of load,the inductive line becomes capacitive gradually and voltage sags become slower,and until the load is reduced to a certain extent,the line voltage starts to rise.

For the front line,installing series capacitor compensation equals to adding a capacitive load at the rear of the line.With the further reduction of loads,line impedance becomes negative,voltage along the line will rise and the voltage curve goes upward.Meanwhile,line voltage drop caused by the ground capacitance of longdistance line start play a major role,the load becomes smaller,the rate of rising voltage increases and the voltage curve goes upward more quickly.For the rear line, when the load is reduced to a certain extent,due to the ground capacitance of long-distance line,the long lines under light load is capacitive,the line voltage climes slowly and it is shows by the slow ascending voltage curve.

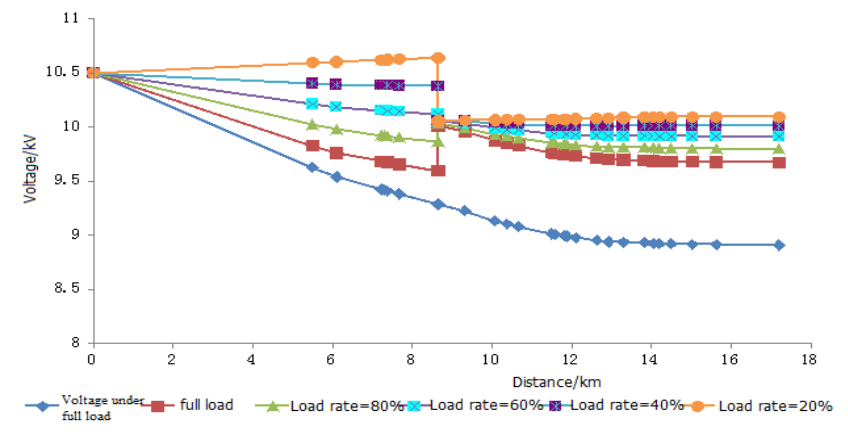

Figure 5 Voltage Distribution of Different LoadRate

In a word,when the load decreases by 30 percent,series compensation device should be removed if do not take other measures to control the voltage.We can take the current at this time as a reference current,monitor the current and remove the capacitor when the amplitude and phase angle of the measured current are close to the reference current.

Actual lines are different as well as their characteristics.we may set different series compensation degree and the reference current may differ too,so $30 \%$ of the load rate indicators do not have universality.For a actual project,only if it is simulated and an analyzed can we obtain valuable result to construct.

\section{Conclusions}

In this paper,the problem of application of series capacitor compensation to $10 \mathrm{kV}$ power distribution network in XiangShan County Shanggang is comprehensively analyzed with digital simulation.And the conclusions are as follows:

(1)To obtain the best technical and economic advantages,we should select appropriate installing location and capacity of series capacitor and choose concentrated compensation or distributed compensation by the actual situation of lines.

(2)Fixed series compensation is self-adaptive to load change and has a compensation effect to heavy load line, but light load line with high load fluctuation may cause abnormal voltage rise in front the compensation point,that needs setting reasonable rules to switch series capacitor.

(3)Since loads vary wildly during day and night in urban and rural distribution network,and some loads may be very small at night,estimated method for position and capacity of series capacitor is advisable and practical.

\section{References}

[1] Rui Cao. Analysis and application research on the current situation of reactive power compensation in distribution network.[D]. North china electric power university, 2011. (in Chinese)

[2] Jingjing Zhang. How to improve the voltage quality of $10 \mathrm{kv}$ power distribution line in substation[J]. Information technology, 2010(9):797-798. (in Chinese) 
[3] Guying Zuo,Daozuo Jiang, Yiqiao Liang, etc. A research of D-FSC for improving voltage quality in distribution networks[J]. Power system protection and control, 2013(08):61-67. (in Chinese)

[4] Yonghai Wu, Jinding Cai. Study and application of voltage regulation technology by series capacitance compensation in medium voltage distribution network[J]. Power system protection and control, 2012(13):140-144. (in Chinese)

[5] Ruju Fang. Power capacitor selection and calculation for serial compensation[A]. Proceedings of China power system protection and control seminar, 2008:598-600. (in Chinese)

[6] Xiaomeng $\mathrm{Wu}$, Jian Liu,Pengxiang Bi. Rearch on voltage stability of distribution networks[J]. Power system technology, 2006(24):31-35 .(in Chinese) 